\title{
A short proof of the Harris-Kesten Theorem
}

\author{
Béla Bollobás*†‡§ Oliver Riordan ${ }^{\dagger \S \rrbracket}$
}

February 1, 2008

\begin{abstract}
We give a short proof of the fundamental result that the critical probability for bond percolation in the planar square lattice $\mathbb{Z}^{2}$ is equal to $1 / 2$. The lower bound was proved by Harris, who showed in 1960 that percolation does not occur at $p=1 / 2$. The other, more difficult, bound was proved by Kesten, who showed in 1980 that percolation does occur for any $p>1 / 2$.
\end{abstract}

\section{Introduction}

Let us set the scene by recalling some basic notions of percolation theory, in the very special context we shall study here; for general background, we refer the reader to Kesten [17, Chayes and Chayes [8] and Grimmett [10]. Let $\mathbb{Z}^{2}$ be the planar square lattice, i.e., the graph whose vertices are the points of $\mathbb{Z}^{2}$, in which vertices at Euclidean distance 1 are joined by an edge. A bond percolation measure on $\mathbb{Z}^{2}$, or any other graph, is a probability measure on the space of assignments of a state, namely open or closed, to each edge $e \in E\left(\mathbb{Z}^{2}\right)$ (with the usual $\sigma$-field of measurable events). Most of the time we shall consider the product measure $\mathbb{P}_{p}=\mathbb{P}_{p}^{\mathbb{Z}^{2}}$, in which the states of the edges are independent, and every edge is open with probability $p$.

An open cluster is a maximal connected subgraph of $\mathbb{Z}^{2}$ all of whose edges are open. We write $C_{v}$ for the open cluster containing a given vertex $v \in \mathbb{Z}^{2}$. Thus a vertex $w$ lies in $C_{v}$ if and only if $w$ can be reached from $v$ by an open path, i.e., a path in $\mathbb{Z}^{2}$ all of whose edges are open.

The fundamental question of percolation theory is 'when does percolation occur', i.e., 'for which $p$ is there an infinite open cluster'? Of course, this

\footnotetext{
*Department of Mathematical Sciences, University of Memphis, Memphis TN 38152, USA

†Trinity College, Cambridge CB2 1TQ, UK

${ }^{\ddagger}$ Research supported in part by NSF grant ITR 0225610 and DARPA grant F33615-01-C1900

$\S$ Research partially undertaken during a visit to the Forschungsinstitut für Mathematik, ETH Zürich

๑ Royal Society Research Fellow, Department of Pure Mathematics and Mathematical Statistics, University of Cambridge, UK
} 
question can be, and is, asked in a wide variety of contexts. Here we shall consider only the particular case of bond percolation in $\mathbb{Z}^{2}$ described above. Note that the question makes sense: if $E_{\infty}$ is the event that there is an infinite open cluster, then by Kolmogorov's $0 / 1$-law, $\mathbb{P}_{p}\left(E_{\infty}\right)$ is 0 or 1 for any $p$.

Writing $\left|C_{v}\right|$ for the number of vertices of $C_{v}$, let

$$
\theta(p)=\mathbb{P}_{p}\left(\left|C_{0}\right|=\infty\right)
$$

where $0=(0,0)$ is the origin. Now $\left|C_{0}\right|=\infty$ implies $E_{\infty}$, while $E_{\infty}$ is the countable union of the events $\left|C_{v}\right|=\infty, v \in \mathbb{Z}^{2}$. From translational invariance we have $\mathbb{P}_{p}\left(\left|C_{v}\right|=\infty\right)=\theta(p)$ for all $v$, so $\mathbb{P}_{p}\left(E_{\infty}\right)$ is 0 if $\theta(p)=0$ and 1 if $\theta(p)>0$; thus, the question of when percolation occurs is precisely the question 'when is $\theta(p)>0$ ?' It is easy to see that $\theta(p)$ is an increasing function of $p$, so there is a 'critical probability'

$p_{H}=p_{H}\left(\mathbb{Z}^{2}\right)=\inf \{p: \theta(p)>0\}=\inf \left\{p: \mathbb{P}_{p}\left(E_{\infty}\right)>0\right\}=\inf \left\{p: \mathbb{P}_{p}\left(E_{\infty}\right)=1\right\}$

Here, following Welsh (see [21]), the $H$ is in honour of Hammersley, although it is more common to write $p_{c}$ for $p_{H}$. In simple cases such as the present one, it is easy to see that $0<p_{H}<1$. The problem of investigating $p_{H}$ in a variety of contexts was posed by Broadbent and Hammersley [7] in 1957. Hammersley 11, 12, 13. proved general results implying in particular that $0.35<$ $p_{H}\left(\mathbb{Z}^{2}\right)<0.65$. The first major progress was due to Harris [14, who proved in 1960 that $p_{H}\left(\mathbb{Z}^{2}\right) \geq 1 / 2$. His proof makes use of the 'self-duality' of $\mathbb{Z}^{2}$, and is highly non-trivial.

For many years it was believed that $p_{H}=1 / 2$ for bond percolation in $\mathbb{Z}^{2}$; see, for example, Sykes and Essam 22. However, it was only in 1980, twenty years after Harris proved that $p_{H} \geq 1 / 2$, that Kesten [16] proved this conjecture, following significant progress by Russo [20] and Seymour and Welsh [21].

The proofs of Harris and Kesten are beautiful, but rather complicated and long. Other proofs of their results have since been developed, for example that due to Zhang (see [10]), and very much more general results are now known; however, none of the proofs is very simple. Here we shall give short proofs of the Harris-Kesten results for $p_{H}\left(\mathbb{Z}^{2}\right)$, the critical probability for bond percolation in $\mathbb{Z}^{2}$. Our methods are applicable in other contexts as well; indeed, they were developed in [4] to prove that the critical probability for random Voronoi percolation in the plane is $1 / 2$.

It has been known for a long time that Kesten's result follows easily once one can show that a certain 'rectangle crossing' event undergoes a 'sharp transition', in that for any $p>1 / 2$ its probability is close to 1 whenever the rectangle is large enough. The key to our method is a simple deduction of this result from a general result of probabilistic combinatorics, due to Friedgut and Kalai [9]. There are two other components to the proof. For the first, we use the strategy of Russo 20] and Seymour and Welsh [21, but give a shorter proof. For the second, deducing percolation from a large rectangle-crossing probability, we give three arguments, one that 'happens to come out' because of how the numbers 
work out, a well-known, elegant 'renormalization' argument, and a more recent argument that is likely to be useful in other contexts.

The rest of the paper is organized as follows. In the next section we present two results from probabilistic combinatorics; with the exception of these results, the arguments to come will be self-contained. In Section 3 we discuss the 'selfduality' of $\mathbb{Z}^{2}$. Harris' Theorem will be proved in Section 4 the key application of the Friedgut-Kalai result will be given in Section 5 and three ways of deducing Kesten's Theorem in Section [ [ In the last section we briefly discuss possible extensions.

\section{Preliminaries}

The first result we shall need is a very simple but fundamental lemma due to Harris 14. Let $X$ be a fixed ground set with $N$ elements, and let $X_{p}$ be a random subset of $X$ obtained by selecting each $x \in X$ independently with probability $p$. For a family $\mathcal{A} \subset \mathcal{P}(X)$ of subsets of $X$, let $\mathbb{P}_{p}^{X}(\mathcal{A})$ be the probability that $X_{p} \in \mathcal{A}$. In this context, $\mathcal{A}$ is increasing if $A \in \mathcal{A}$ and $A \subset B \subset X$ imply $B \in \mathcal{A}$. For example, $X$ might be a finite set of edges of $\mathbb{Z}^{2}$, and $X_{p}$ the subset of $X$ consisting of the open edges. Then an event is increasing if it is preserved when the states of one or more edges are changed from closed to open.

Lemma 1. If $\mathcal{A}, \mathcal{B} \subset \mathcal{P}(X)$ are increasing, then for any $p$ we have

$$
\mathbb{P}_{p}^{X}(\mathcal{A} \cap \mathcal{B}) \geq \mathbb{P}_{p}^{X}(\mathcal{A}) \mathbb{P}_{p}^{X}(\mathcal{B}) .
$$

In other words, increasing events are positively correlated. Once one thinks of the statement, the proof turns out to be very simple, using induction on $N$. Taking complements, Lemma 1 also applies to two decreasing events, defined in the obvious way; similarly, an increasing event and a decreasing one are negatively correlated. The extension to infinite product spaces is immediate; we shall not need it here. Harris discovered this lemma precisely in the context of percolation, although it is perhaps one of the most basic results of combinatorial probability. In the latter context Harris' Lemma was rediscovered by Kleitman [18; a series of important generalizations then followed, culminating in the very general 'Four-functions Theorem' of Ahlswede and Daykin [1].

The second result we shall need is a sharp-threshold theorem of Friedgut and Kalai 9, which is itself a simple consequence of a result of Kahn, Kalai and Linial [15] (see also [6]) concerning the influences of coordinates in a product space.

With the same set-up as above, $\mathcal{A} \subset \mathcal{P}(X)$ is symmetric if there is a permutation group acting transitively on $X$ whose action on $\mathcal{P}(X)$ preserves $\mathcal{A}$. In our notation, the result of Friedgut and Kalai we shall need is as follows.

Theorem 2. There is an absolute constant $c_{1}$ such that if $|X|=N, \mathcal{A} \subset \mathcal{P}(X)$ is symmetric and increasing, $0<\varepsilon<1 / 2$, and $\mathbb{P}_{p}^{X}(\mathcal{A})>\varepsilon$, then $\mathbb{P}_{q}^{X}(\mathcal{A})>1-\varepsilon$ 
whenever

$$
q-p \geq c_{1} \frac{\log (1 /(2 \varepsilon))}{\log N} .
$$

Having collected the basic results above for future reference, we are ready to start our simple proof that $p_{H}\left(\mathbb{Z}^{2}\right)=1 / 2$.

\section{Self-duality}

Throughout this paper we shall work with 'open crossings of rectangles', a standard concept of planar percolation. We shall identify a rectangle $R=$ $[a, b] \times[c, d]$, where $a<b$ and $c<d$ are integers, with an induced subgraph of $\mathbb{Z}^{2}$. This subgraph includes all vertices and edges in the interior and boundary of $R$. If $b-a=k$ and $d-c=\ell$ then we call $R$ a $k$ by $\ell$ rectangle.

We write $H(R)$ for the event that there is a horizontal open crossing of $R$, i.e., a path from the left side of $R$ to the right side consisting entirely of open edges of $R$. Similarly, we write $V(R)$ for the event that there is a vertical open crossing of $R$. Note that for disjoint rectangles $R$ and $R^{\prime}$ (a rectangle includes its boundary) each of the events $H(R), V(R)$ is independent of each of the events $H\left(R^{\prime}\right), V\left(R^{\prime}\right)$.

The dual $L^{*}$ of the lattice $L=\mathbb{Z}^{2}$ is the usual planar dual of $\mathbb{Z}^{2}$ considered as a graph drawn in the plane: there is a vertex $v \in L^{*}$ corresponding to each face of $\mathbb{Z}^{2}$, i.e., to each square $[x, x+1] \times[y, y+1], x, y \in \mathbb{Z}$. It is customary to take $v=(x+1 / 2, y+1 / 2)$. There is one edge $e^{*}$ of $L^{*}$ corresponding to each edge $e$ of $L$; this edge joins the two vertices of $L^{*}$ corresponding to the faces of $L$ in whose boundary $e$ lies. Of course, $L^{*}$ may be defined analogously for any plane graph. When $L=\mathbb{Z}^{2}$, then $L^{*}=(1 / 2,1 / 2)+\mathbb{Z}^{2}$ is isomorphic to $L$, as shown below.

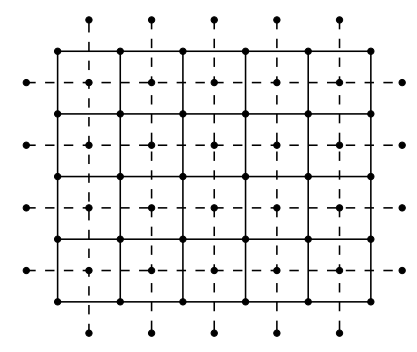

Figure 1: Portions of the lattice $L=\mathbb{Z}^{2}$ (solid lines) and the isomorphic dual lattice $L^{*}$ (dashed lines).

The horizontal dual, or simply the dual, of a rectangle $R=[a, b] \times[c, d]$ is the rectangle $R^{h}=[a+1 / 2, b-1 / 2] \times[c-1 / 2, d+1 / 2]$ in $L^{*}$. Defining an edge $e^{*}$ of $L^{*}$ to be open if and only if $e$ is closed, let $V^{*}\left(R^{h}\right)$ be the event that there is a vertical crossing of $R^{h}$ by open edges of $L^{*}$. 
The next lemma is key to the analysis of bond percolation in $\mathbb{Z}^{2}$. Often, it is stated as 'obvious' and no formal proof is given. While the result is indeed obvious, it is not entirely trivial to prove. However, a short proof is possible, and, as it happens, needs no topology. For a topological proof of a related result see Kesten [17. pp. 386-392].

Lemma 3. Let $R$ be a rectangle in $L=\mathbb{Z}^{2}$. Whatever the states of the edges in $R$, exactly one of the events $H(R)$ and $V^{*}\left(R^{h}\right)$ holds.

Proof. To avoid fractions, for this proof let $L=(0,2)+4 \mathbb{Z}^{2}$ and $L^{*}=(2,0)+4 \mathbb{Z}^{2}$; also, let $R \subset L$ have vertex set $\{(4 i, 4 j+2): 0 \leq i \leq k, 0 \leq j \leq \ell-1\}$, so that the vertex set of $R^{h}$ is $\{(4 i+2,4 j): 0 \leq i \leq k-1,0 \leq j \leq \ell\}$. The open edges of $R$ form a graph $G$, and those of $R^{h}$ form a graph $G^{h}$. Our task is to show that either $G$ contains a left-right path, i.e., a path from a vertex $(0,4 j+2)$ to a vertex $(4 k, 4 h+2)$, or else $G^{h}$ contains a top-bottom path, but not both. In proving this assertion, we may assume that $G$ contains all $2(\ell-1)$ edges on the left and right sides, and $G^{h}$ contains all 2(k-1) edges at the top and bottom, as in Figure 2 Note that $G$ and $G^{h}$ are plane graphs with every edge a straight-line segment of length 4 .

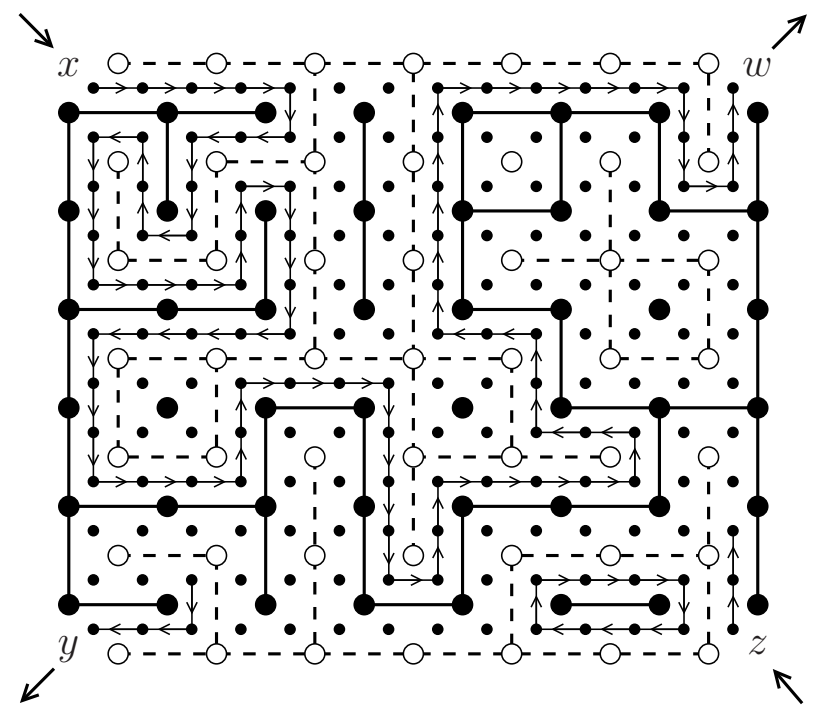

Figure 2: The graphs $G$ (large solid circles and solid lines) and $G^{h}$ (empty circles and dashed lines), and parts of the oriented graph $M$ (small circles and arrows). The part of $M$ shown includes all of the path $P$ and a cyclic component.

We shall construct a third graph, $M$, which is in the 'middle', between $G$ and $G^{h}$. Let $R_{\text {odd }}$ be the $2 k \times 2 \ell$ rectangle with vertex set $\{(2 i+1,2 j+1)$ : $0 \leq i \leq 2 k-1,0 \leq j \leq 2 \ell-1\}$, and let $M$ be the subgraph of $R_{\text {odd }}$ formed by the edges not meeting either an edge of $G$ or an edge of $G^{h}$. Orient every edge of $M$ so that $G$ is on the right and $G^{h}$ is on the left. Then every vertex of 
$M$ has one edge coming in and one going out, apart from exactly four vertices of degree one, $x, y, z$ and $w$, say, as in Figure 2 Thus the component of $x$ in $M$ is a path $P$ ending at one of $y$ and $w$. If $P$ ends at $w$ then the edges of $G$ to the right of $P$ form a connected subgraph of $G$ meeting the left side and the right side (as in Figure 22), and if $P$ ends at $y$ then the edges of $G^{h}$ to the left of $P$ form a connected subgraph of $G^{h}$ meeting the top and the bottom. Since a connected subgraph meeting two opposite sides contains a path meeting the same sides, we have shown that at least one of $H(R)$ and $V^{*}\left(R^{h}\right)$ holds.

That $H(R)$ and $V^{*}\left(R^{h}\right)$ cannot both hold is immediate from the Jordan Curve Theorem; however, only the easy part of this theorem is needed. Indeed, if the path $P$ ends at $w$ then $P$ may be completed to a simple closed curve $\bar{P}$ by coming back from $w$ to $x$ over the top of $R^{h}$. This curve winds once around any top vertex of $R^{h}$, and zero times around any bottom vertex. As the winding number of $\bar{P}$ is constant off $\bar{P}$, any top-bottom path in $G^{h}$ must cross $\bar{P}$, and hence cross an edge of $M$, contradicting the definition of $M$. Similarly, if $P$ ends at $y$ there is no left-right path in $G$, completing the proof.

As the edges of $L^{*}$ are open independently with probability $1-p$, Lemma 3 has the following immediate corollary concerning rectangles in the original lattice $L=\mathbb{Z}^{2}$.

Corollary 4. If $R$ is a $k$ by $\ell-1$ rectangle in $\mathbb{Z}^{2}$ and $R^{\prime}$ is a $k-1$ by $\ell$ rectangle, then

$$
\mathbb{P}_{p}(H(R))+\mathbb{P}_{1-p}\left(V\left(R^{\prime}\right)\right)=1 .
$$

If $k=\ell=n+1$, then $R^{\prime}$ is $R$ rotated by 90 degrees. Thus $\mathbb{P}_{p}(H(R))=$ $\mathbb{P}_{p}\left(V\left(R^{\prime}\right)\right)$, so Corollary $\square$ implies the following essential consequence of the self-duality of $\mathbb{Z}^{2}$.

Corollary 5. If $R$ is an $n+1$ by $n$ rectangle then $\mathbb{P}_{1 / 2}(H(R))=1 / 2$. Thus, if $S$ is an $n$ by $n$ square, then

$$
\mathbb{P}_{1 / 2}(V(S))=\mathbb{P}_{1 / 2}(H(S)) \geq 1 / 2 .
$$

It is easy to think that Corollary 5 shows that $p_{H}=1 / 2$. Although selfduality is of course the reason 'why' $p_{H}=1 / 2$, a rigorous deduction is far from easy, and took twenty years to accomplish.

\section{A short proof of Harris' Theorem}

In this section we present a short proof of Harris' 1960 result that $\theta(1 / 2)=0$, and hence $p_{H}=p_{H}\left(\mathbb{Z}^{2}\right) \geq 1 / 2$. The techniques are considerably simpler than Harris' original ones. The strategy of the proof is that of Russo 20] and Seymour and Welsh [21]; however, our proof of the key intermediate result is considerably shorter than theirs. 
Lemma 6. Let $R=[0, m] \times[0,2 n], m \geq n$, be an $m$ by $2 n$ rectangle. Let $X(R)$ be the event that there are paths $P_{1}$ and $P_{2}$ of open edges, where $P_{1}$ crosses $S=[0, n] \times[0, n]$ from top to bottom, and $P_{2}$ lies inside $R$ and joins some vertex on $P_{1}$ to some vertex on the right-hand side of $R$. Then $\mathbb{P}_{p}(X(R)) \geq$ $\mathbb{P}_{p}(H(R)) \mathbb{P}_{p}(V(S)) / 2$.

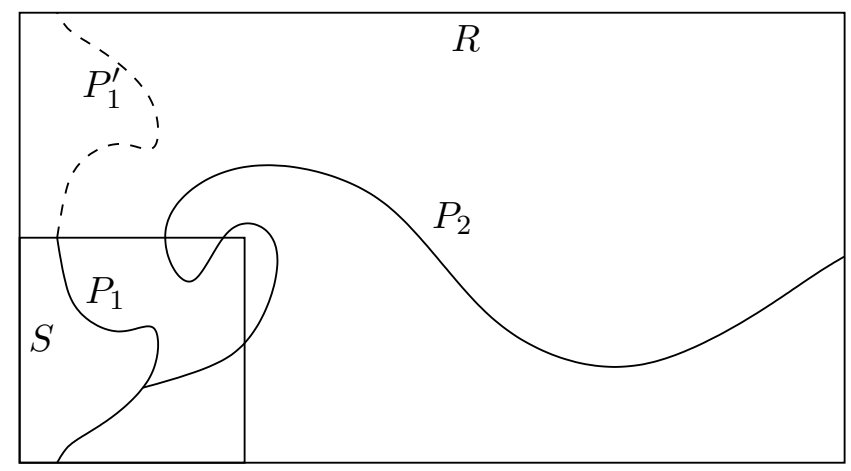

Figure 3: A rectangle $R$ and square $S$ inside it, drawn with paths (solid curves) whose presence as open paths would imply $X(R)$.

Proof. Suppose that $V(S)$ holds, so there is a path $P$ of open edges crossing $S$ from top to bottom. Note that any such $P$ separates $S$ into two pieces, one to the left of $P$ and one to the right. The proof of Lemma 3 shows that when $V(S)$ holds, one can define the left-most such path $P, L V(S)$, in such a way that the event $\left\{L V(S)=P_{1}\right\}$ does not depend on edges to the right of $P_{1}$.

It is easy to see that for any possible value $P_{1}$ of $L V(S)$ we have

$$
\mathbb{P}_{p}\left(X(R) \mid L V(S)=P_{1}\right) \geq \mathbb{P}_{p}(H(R)) / 2:
$$

let $P$ be the (not necessarily open) path formed by the union of $P_{1}$ and its reflection $P_{1}^{\prime}$ in the line $y=n$; see Figure 3 This path crosses $[0, n] \times[0,2 n]$ from top to bottom. With (unconditional) probability $\mathbb{P}_{p}(H(R))$ there is a path $P_{3}$ of open edges crossing $R$ from right to left - this path must meet $P$. By symmetry, the (unconditional) probability that some such path first meets $P$ at a point of $P_{1}$ is at least $\mathbb{P}_{p}(H(R)) / 2$. Hence, the event $Y\left(P_{1}\right)$ that there is a path $P_{2}$ in $R$ to the right of $P$ joining some point of $P_{1}$ to the right-hand side of $R$ has probability at least $\mathbb{P}_{p}(H(R)) / 2$. But $Y\left(P_{1}\right)$ depends only on edges to the right of $P$. All such edges in $S$ are to the right of $P_{1}$ in $S$. As the states of these edges are independent of $\left\{L V(S)=P_{1}\right\}$, we have

$$
\mathbb{P}_{p}\left(Y\left(P_{1}\right) \mid L V(S)=P_{1}\right)=\mathbb{P}_{p}\left(Y\left(P_{1}\right)\right) \geq \mathbb{P}_{p}(H(R)) / 2 .
$$

But $L V(S)=P_{1}$ and $Y\left(P_{1}\right)$ imply $X(R)$, so we have $\mathbb{P}_{p}\left(X(R) \mid L V(S)=P_{1}\right) \geq$ $\mathbb{P}_{p}(H(R)) / 2$. Finally, the event $V(S)$ is a disjoint union of events of the form $\left\{L V(S)=P_{1}\right\}$, so $\mathbb{P}_{p}(X(R) \mid V(S)) \geq \mathbb{P}_{p}(H(R)) / 2$. 
The following immediate corollary of Lemma 6 is standard, but is usually proved in a different way (by the methods of Russo [20] or Seymour and Welsh [21]; see [10]).

Corollary 7. Let $\rho>1$ be a fixed integer. There is a constant $c_{2}(\rho)>0$ depending only on $\rho$ such that for any $2 \rho n$ by $2 n$ rectangle $R$ we have $\mathbb{P}_{1 / 2}(H(R)) \geq$ $c_{2}(\rho)$.

Note that we take $\rho$ to be an integer only because we want all our rectangles to have integer coordinates.

Proof. Writing $h_{m, n}$ for $\mathbb{P}_{1 / 2}(H(R))$, where $R$ is an $m$ by $n$ rectangle, we claim that for $m \geq n$ we have $h_{2 m-n, 2 n} \geq h_{m, 2 n}^{2} / 2^{5}$. Applying this repeatedly, starting from $h_{2 n, 2 n} \geq 1 / 2$ (by Corollary 5 ), we obtain the result.

To see the claim, consider the rectangles $R=[0, m] \times[0,2 n], R^{\prime}=[n-$ $m, n] \times[0,2 n]$ and the square $S=[0, n] \times[0, n]$ in their intersection, as shown in Figure 4

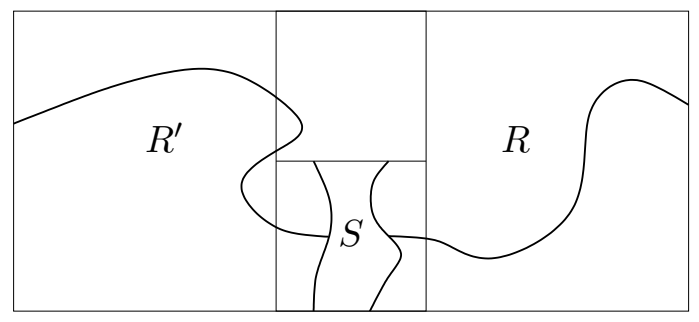

Figure 4: The overlapping rectangles $R$ and $R^{\prime}$ with the square $S$ in their intersection. The paths drawn show that $X(R)$ holds, as well as the reflected equivalent for $R^{\prime}$. If $H(S)$ also holds, then so does $H\left(R \cup R^{\prime}\right)$.

If $E_{1}=X(R)$, the corresponding (i.e., horizontally reflected) event $E_{2}$ for $R^{\prime}$, and $E_{3}=H(S)$ all hold, then so does $H\left(R \cup R^{\prime}\right)$, using only the fact that any horizontal crossing of $S$ meets any vertical crossing. (See Figure 4 ) But the $E_{i}$ are increasing events, so, by Lemma 1

$$
\mathbb{P}_{1 / 2}\left(H\left(R \cup R^{\prime}\right)\right) \geq \mathbb{P}_{1 / 2}\left(E_{1}\right) \mathbb{P}_{1 / 2}\left(E_{2}\right) \mathbb{P}_{1 / 2}\left(E_{3}\right)=\mathbb{P}_{1 / 2}(X(R))^{2} \mathbb{P}_{1 / 2}(H(S)) .
$$

By Corollary $\left[\mathbb{P}_{1 / 2}(V(S))=\mathbb{P}_{1 / 2}(H(S)) \geq 1 / 2\right.$. Applying Lemma $[$ it follows that

$$
\begin{aligned}
\mathbb{P}_{1 / 2}\left(H\left(R \cup R^{\prime}\right)\right) & \geq \mathbb{P}_{1 / 2}(H(R))^{2} \mathbb{P}_{1 / 2}(V(S))^{2} \mathbb{P}_{1 / 2}(H(S)) / 4 \\
& \geq \mathbb{P}_{1 / 2}(H(R))^{2} / 2^{5}
\end{aligned}
$$

This proves the claim and thus the corollary.

As noted by Russo [20] and Seymour and Welsh [21], Harris' result is an easy consequence of Corollary 7 
Theorem 8. For bond percolation in $\mathbb{Z}^{2}, \theta(1 / 2)=0$.

Proof. Let $c_{2}=c_{2}(3)$ be the absolute constant given by Corollary 7 By Corollary 17 and Lemma 10 if we arrange four $6 n$ by $2 n$ rectangles to form a 'square annulus' as in Figure [ then with probability at least $c_{2}^{4}$ each rectangle is

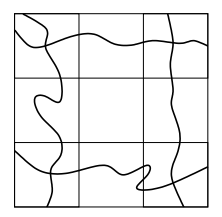

Figure 5: Four rectangles forming a square annulus.

crossed the long way by an open path. The union of any such paths contains a cycle surrounding the centre of the annulus; see Figure 5 For $k \geq 1$, let $A_{k}$ be the square annulus centred on the origin with inner and outer radii $4^{k}$ and $3 \times 4^{k}$. Then the $A_{k}$ (including their boundaries) are disjoint. Using independence of disjoint regions, at $p=1 / 2$ the probability that the point $(1 / 2,1 / 2)$ is not surrounded by a cycle of open edges is at most

$$
\prod_{k=1}^{\infty} \mathbb{P}_{1 / 2}\left(A_{k} \text { contains no open cycle }\right) \leq \prod_{k=1}^{\infty}\left(1-c_{2}^{4}\right)=0 .
$$

Hence, at $p=1 / 2$, the probability that $(1 / 2,1 / 2)$ is in an infinite open cluster in the dual lattice is zero. Equivalently, at $p=1 / 2$ the probability that there is an infinite open cluster is 0 .

\section{Crossing rectangles with high probability}

Surprisingly, although it was twenty years after Harris proved that $p_{H}\left(\mathbb{Z}^{2}\right) \geq 1 / 2$ that Kesten found an ingenious proof that $p_{H}\left(\mathbb{Z}^{2}\right)=1 / 2$, one can use the results mentioned in Section 2 to deduce Kesten's Theorem from Corollary 7 with very little additional work. The main idea is to find a way of using Theorem 2 to show that large rectangles can be crossed with probability close to 1 .

Lemma 9. Let $p>1 / 2$ and an integer $\rho>1$ be fixed. There are constants $\delta=\delta(p)>0$ and $n_{0}=n_{0}(p, \rho)$ such that, if $n \geq n_{0}$ and $R_{n}$ is a $4 \rho n$ by $4 n$ rectangle in $\mathbb{Z}^{2}$, then $\mathbb{P}_{p}\left(H\left(R_{n}\right)\right) \geq 1-n^{-\delta}$.

Proof. To simplify the notation we shall prove the lemma in the case $\rho=3$, which immediately implies the case $\rho=2$; these are the only cases we shall use. The full result can be proved in the same way; it also follows from the case $\rho=2$, say, by combining crossings as in Figure $\mathbf{7}$

Fix $p>1 / 2$. From Corollary 7 applied with $\rho=7$, there is an absolute constant $c_{2}, 0<c_{2}<1 / 2$, such that $\mathbb{P}_{1 / 2}(H(R)) \geq c_{2}$ for any $14 n$ by $2 n$ rectangle 
$R$. If $H(R)$ were a symmetric event then we could just apply Theorem 2 but $H(R)$ is not symmetric, so we shall introduce symmetric auxiliary events.

For $n \geq 3$, let $\mathbb{T}_{n}$ be the $n$ by $n$ discrete torus, i.e., the graph $C_{n} \times C_{n}$, which may be obtained from $\mathbb{Z}^{2}$ by identifying all pairs of vertices for which the corresponding coordinates are congruent modulo $n$. Thus $\mathbb{T}_{n}$ has $n^{2}$ vertices and $2 n^{2}$ edges. For $1 \leq k, \ell \leq n-2$, a $k$ by $\ell$ rectangle $R$ in $\mathbb{T}_{n}$ is an induced subgraph of $\mathbb{T}_{n}$ corresponding to a $k$ by $\ell$ rectangle $R^{\prime}=[a, a+k] \times[b, b+\ell]$ in $\mathbb{Z}^{2}$. Note that our rectangles in the torus are always too small to 'wrap around', so the induced subgraph of $\mathbb{T}_{n}$ is isomorphic to the corresponding induced subgraph of $\mathbb{Z}^{2}$.

We shall take the edges of $\mathbb{T}_{n}$ to be open independently with probability $p$, writing $\mathbb{P}_{p}^{\mathbb{T}_{n}}$ for the corresponding probability measure. Most of the time, we shall suppress the dependence on $n$.

Let $E_{n}$ be the event that $\mathbb{T}_{16 n}$ contains some $14 n$ by $2 n$ rectangle with a horizontal crossing by open edges, or some $2 n$ by $14 n$ rectangle with a vertical crossing. Note that $E_{n}$ is symmetric as a subset of $\mathcal{P}(X)$, where $X$ is the edge set of $\mathbb{T}_{16 n}$, which has cardinality $N=512 n^{2}$.

Considering one fixed $14 n$ by $2 n$ rectangle $R$ in the torus, which we may identify with a corresponding rectangle in $\mathbb{Z}^{2}$, and writing $\mathbb{T}$ for $\mathbb{T}_{16 n}$, we have

$$
\mathbb{P}_{1 / 2}^{\mathbb{T}}\left(E_{n}\right) \geq \mathbb{P}_{1 / 2}^{\mathbb{T}}(H(R))=\mathbb{P}_{1 / 2}(H(R)) \geq c_{2} .
$$

Let $\delta=(p-1 / 2) /\left(64 c_{1}\right)$, where $c_{1}$ is the constant in Theorem 2 and set $\varepsilon=$ $n^{-128 \delta}$. As $\delta$ depends only on $p$, there is an $n_{0}=n_{0}(p)$ such that $\varepsilon<c_{2} \leq 1 / 2$ for all $n \geq n_{0}$. Now

$$
p-\frac{1}{2}=64 c_{1} \delta=c_{1} \frac{\log (1 / \varepsilon)}{\log \left(n^{2}\right)}>c_{1} \frac{\log (1 /(2 \varepsilon))}{\log \left(512 n^{2}\right)} .
$$

Hence, by Theorem 2 as $\mathbb{P}_{1 / 2}^{\mathbb{T}}\left(E_{n}\right) \geq c_{2}>\varepsilon$, we have

$$
\mathbb{P}_{p}^{\mathbb{T}}\left(E_{n}\right) \geq 1-\varepsilon=1-n^{-128 \delta}
$$

for all $n \geq n_{0}$.

Clearly, there are $12 n$ by $4 n$ rectangles $R_{1}, \ldots, R_{64}$ covering $\mathbb{T}=\mathbb{T}_{16 n}$ such that any $14 n$ by $2 n$ rectangle $R$ in $\mathbb{T}$ crosses one of the $R_{i}$, in the sense that the intersection of $R$ and $R_{i}$ is a $12 n$ by $2 n$ subrectangle of $R_{i}$. For example, we may take the $12 n$ by $4 n$ rectangles $R_{i}$ whose bottom-left coordinates are all possible multiples of $2 n$. Similarly, there are $4 n$ by $12 n$ rectangles $R_{65}, \ldots, R_{128}$ so that any $2 n$ by $14 n$ rectangle in $\mathbb{T}$ crosses one of these from top to bottom.

It follows that if $E_{n}$ holds, then so does one of the events $E_{n, i}, i=1, \ldots, 128$, where each $E_{n, i}$ is the event that $R_{i}$ is crossed the long way by an open path. Thus $E_{n}^{c}$, the complement of $E_{n}$, contains the intersection of the $E_{n, i}^{c}$.

By Lemma [1] applied to the product measure $\mathbb{P}_{p}^{\mathbb{T}}$, for each $i$ the decreasing events $E_{n, i}^{c}$ and $\cap_{j<i} E_{n, j}^{c}$ are positively correlated. Hence

$$
\mathbb{P}_{p}^{\mathbb{T}}\left(E_{n}^{c}\right) \geq \mathbb{P}_{p}^{\mathbb{T}}\left(\bigcap_{i=1}^{128} E_{n, i}^{c}\right) \geq \prod_{i=1}^{128} \mathbb{P}_{p}^{\mathbb{T}}\left(E_{n, i}^{c}\right)=\mathbb{P}_{p}^{\mathbb{T}}\left(E_{n, 1}^{c}\right)^{128} .
$$


Thus, from (2), for $n \geq n_{0}$ we have

$$
\mathbb{P}_{p}^{\mathbb{T}}\left(E_{n, 1}^{c}\right) \leq \mathbb{P}_{p}^{\mathbb{T}}\left(E_{n}^{c}\right)^{1 / 128} \leq n^{-\delta},
$$

so $\mathbb{P}_{p}^{\mathbb{T}}\left(E_{n, 1}\right) \geq 1-n^{-\delta}$.

Now $E_{n, 1}$ is the event that there is a horizontal open crossing of a fixed $12 n$ by $4 n$ rectangle $R$ in the torus, which we may identify with a corresponding rectangle in $\mathbb{Z}^{2}$. Thus

$$
\mathbb{P}_{p}(H(R))=\mathbb{P}_{p}^{\mathbb{T}_{16 n}}(H(R)) \geq 1-n^{-\delta}
$$

whenever $R$ is a $12 n$ by $4 n$ rectangle in $\mathbb{Z}^{2}$ and $n$ is large enough, completing the proof of the lemma.

\section{Three ways of deducing Kesten's Theorem}

Our first deduction makes use of the quantitative form of Lemma 9 Recall that $E_{\infty}$ is the event that there is an infinite open cluster, and that $\mathbb{P}_{p}\left(E_{\infty}\right)>0$ implies that $\mathbb{P}_{p}\left(E_{\infty}\right)=1$ and $\theta(p)>0$.

Theorem 10. For bond percolation in $\mathbb{Z}^{2}$, if $p>1 / 2$ then $\mathbb{P}_{p}\left(E_{\infty}\right)=1$.

Proof of Theorem 10-first version. Fix $p>1 / 2$. Let $\delta=\delta(p)$ and $n_{0}=$ $n_{0}(p, 2)$ be as in Lemma 9 Let $m \geq n_{0}$ be an integer to be chosen below, and set $n=4 m$. For $k=0,1,2, \ldots$, let $R_{k}$ be a rectangle with bottom-left corner the origin and side-lengths $2^{k} n$ and $2^{k+1} n$, where the longer side is vertical if $k$ is even and horizontal if $k$ is odd; see Figure 6

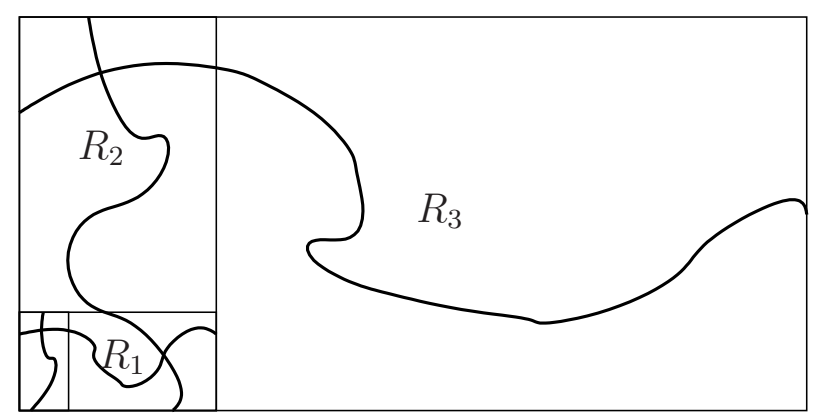

Figure 6: The rectangles $R_{0}$ to $R_{3}$ ( $R_{0}$ not labelled) drawn with open paths corresponding to the events $E_{k}$.

Let $E_{k}$ be the event that $R_{k}$ is crossed the long way by an open path. Note that any two such crossings of $R_{k}$ and $R_{k+1}$ must meet, so if all the $E_{k}$ hold, then so does $E_{\infty}$. If $n$ is large enough then, by Lemma 9

$$
\sum_{k \geq 0} \mathbb{P}_{p}\left(E_{k}^{c}\right) \leq \sum_{k \geq 0}\left(2^{k} n\right)^{-\delta}=\frac{n^{-\delta}}{1-2^{-\delta}}<1,
$$


so $\mathbb{P}_{p}\left(E_{\infty}\right) \geq \mathbb{P}_{p}\left(\bigcap_{k \geq 0} E_{k}\right)>0$.

Together, Theorems 8 and 10 show that $p_{H}\left(\mathbb{Z}^{2}\right)=1 / 2$.

The argument above depends on the result of a certain calculation: it is important that the sum of the 'error probabilities' $n^{-\delta}$ as $n$ runs over powers of 2 is convergent. It might appear that the basic strategy of the proof thus depends on the serendipitous form of the bound on $q-p$ in (10). In fact, this is not the case: one only needs the qualitative result that for each $\varepsilon>0$, the bound tends to zero as $n \rightarrow \infty$. Starting from this weaker result, the argument of Section 5 implies the following qualitative form of Lemma 9

Lemma 11. Let $p>1 / 2$ be fixed. If $R_{n}$ is a $3 n$ by $n$ rectangle in $\mathbb{Z}^{2}$, then $\mathbb{P}_{p}\left(H\left(R_{n}\right)\right) \rightarrow 1$ as $n \rightarrow \infty$.

It is well known that this lemma implies Kesten's Theorem. We give two arguments. The first is a 'renormalization' argument due to Aizenman, Chayes, Chayes, Fröhlich and Russo [2]; see also Chayes and Chayes [8].

Proof of Theorem [10 - second version. Let us call a crossing of a rectangle internal if it uses no edges in the boundary of the rectangle. Fixing $p>1 / 2$ as before, let $i_{n}$ be the probability that a $2 n$ by $n$ rectangle has an internal horizontal open crossing. Note that $i_{n}$ is the probability that a $2 n$ by $n-2$ rectangle has a horizontal open crossing.

Writing $s_{n}$ for the probability that an $n$ by $n$ square has an internal vertical open crossing, as an $n$ by $n$ square is contained in an $n$ by $2 n$ rectangle, $s_{n} \geq i_{n}$. Considering three $2 n$ by $n$ rectangles overlapping in two $n$ by $n$ squares as in Figure 7 by Lemma 10 probability that a $4 n$ by $n$ rectangle has an internal horizontal open crossing is at least $i_{n}^{3} s_{n}^{2}$. Placing two such rectangles side by side to form a $4 n$ by $2 n$ rectangle, the events that each has an internal open crossing depend on disjoint sets of edges and are thus independent. Hence,

$$
i_{2 n} \geq 1-\left(1-i_{n}^{3} s_{n}^{2}\right)^{2} \geq 1-\left(1-i_{n}^{5}\right)^{2} .
$$

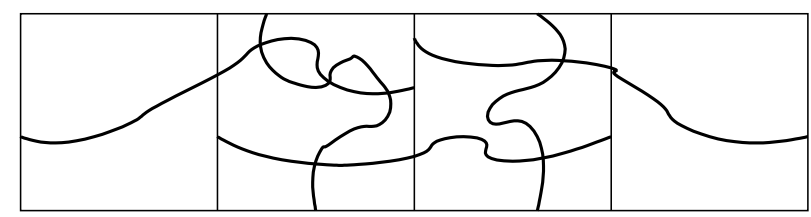

Figure 7: Three $2 n$ by $n$ rectangles and two $n$ by $n$ squares, drawn with paths guaranteeing a horizontal open crossing of their union

Writing $i_{n}$ as $1-\varepsilon$, from (4) we have $i_{2 n} \geq 1-25 \varepsilon^{2}$, which is at least $1-\varepsilon / 2$ if $\varepsilon \leq 1 / 50$. By Lemma [1] there is an $n$ with $i_{n} \geq 0.98$. (If $n \geq 6$ then a 
crossing of a fixed $3(n-2)$ by $n-2$ rectangle includes an internal crossing of a fixed $2 n$ by $n$ rectangle.) It follows that $i_{2^{k} n} \geq 1-2^{-k} / 50$, and $\mathbb{P}_{p}\left(E_{\infty}\right)>0$ follows as in the first proof of Theorem 10.

This method shows that if for a single value of $n$ we have $i_{n}>0.951 \ldots$ (a root of $\left.x=1-\left(1-x^{5}\right)^{2}\right)$, then percolation occurs.

In fact, arguing as in Chayes and Chayes [8, one can do a little better. Note that $s_{n}$ is also the probability that an $n$ by $n$ square has an internal horizontal open crossing. Dividing a $2 n$ by $n$ rectangle into two squares, if the rectangle has an internal horizontal open crossing, so do both squares. As the internal edges of the squares are disjoint, it follows that $i_{n} \leq s_{n}^{2}$. Therefore the first inequality in (4) implies $i_{2 n} \geq 1-\left(1-i_{n}^{4}\right)^{2}$, and the value $0.951 \ldots$ may be replaced by $0.920 \ldots$, a root of $x=1-\left(1-x^{4}\right)^{2}$.

The second argument requires an even weaker initial bound on $i_{n}$; for this we shall need an observation concerning $k$-dependent percolation. A bond percolation measure on $\mathbb{Z}^{2}$ is $k$-dependent if for every pair $S, T$ of sets of edges of $\mathbb{Z}^{2}$ at graph distance at least $k$, the states (being open or closed) of the edges in $S$ are independent of the states of the edges in $T$. When $k=1$, the separation condition is exactly that no edge of $S$ shares a vertex with an edge of $T$.

In static renormalization arguments, $k$-dependent probability measures arise very naturally. Comparisons between such measures and product measures (or arguments amounting to such comparisons) have been considered by several authors; see Liggett, Schonmann and Stacey [19] and the references therein.

Lemma 12. There is a $p_{0}<1$ such that in any 1-dependent bond percolation measure on $\mathbb{Z}^{2}$ satisfying the additional condition that each edge is open with probability at least $p_{0}$, the probability that $\left|C_{0}\right|=\infty$ is positive.

In many contexts the value of $p_{0}$ is important. The best bound known is the result that one can take $p_{0}=0.8639$; this was proved by Balister, Bollobás and Walters [3], who used it in the study of random geometric graphs. For proving Kesten's Theorem, however, the value of $p_{0}$ is not important.

Lemma 12 is an immediate consequence of the very general main result of 19 but, in the form above, is trivial from first principles. Indeed, if $C_{0}$ is finite, then it is surrounded by an open cycle in the dual lattice $L^{*}$. Very crudely, there are at most $\ell 3^{\ell}$ cycles of length $\ell$ in $L^{*}$ surrounding the origin. For each, the corresponding set $S$ of edges of $L=\mathbb{Z}^{2}$ contains a subset $S^{\prime}$ of size at least $|S| / 4=\ell / 4$ in which any two edges are vertex disjoint. By 1-dependence, the states of the edges in $S^{\prime}$ are independent, so the probability that all these edges are closed in $L$ (and thus open in $L^{*}$ ) is at most $\left(1-p_{0}\right)^{\ell / 4}$. Combining these observations shows that $\mathbb{P}\left(\left|C_{0}\right|<\infty\right) \leq \sum_{\ell \geq 4} \ell 3^{\ell}\left(1-p_{0}\right)^{\ell / 4}$, which is less than 1 if $p_{0}$ is large enough.

Proof of Theorem 10 - third version. Let $p>1 / 2$ be fixed, let $p_{0}<1$ be a constant for which Lemma 12 holds, and set $c=p_{0}^{1 / 3}$. Given a $3 n$ by $n$ rectangle 
$R$, let $S^{\prime}$ and $S^{\prime \prime}$ be the two end squares when $R$ is cut into three squares. Note that $H(R)$ certainly implies $H\left(S^{\prime}\right)$ so, by Lemma 11

$$
\mathbb{P}_{p}\left(V\left(S^{\prime \prime}\right)\right)=\mathbb{P}_{p}\left(V\left(S^{\prime}\right)\right)=\mathbb{P}_{p}\left(H\left(S^{\prime}\right)\right) \geq \mathbb{P}_{p}(H(R)) \geq c
$$

if $n$ is large enough, which we shall assume from now on.

Let $G(R)$ be the event $H(R) \cap V\left(S^{\prime}\right) \cap V\left(S^{\prime \prime}\right)$; see Figure 8

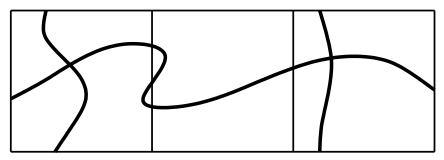

Figure 8: A $3 n$ by $n$ rectangle $R$ such that $G(R)$ holds.

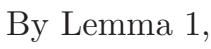

$$
\mathbb{P}_{p}(G(R)) \geq \mathbb{P}_{p}(H(R)) \mathbb{P}_{p}\left(V\left(S^{\prime}\right)\right) \mathbb{P}_{p}\left(V\left(S^{\prime \prime}\right)\right) \geq c^{3}=p_{0} .
$$

Define $G\left(R^{\prime}\right)$ similarly for an $n$ by $3 n$ rectangle, so $\mathbb{P}_{p}\left(G\left(R^{\prime}\right)\right)=\mathbb{P}_{p}(G(R)) \geq p_{0}$.

Let us define a 1-dependent bond percolation measure $\widetilde{\mathbb{P}}$ on $\mathbb{Z}^{2}$ as follows: the edge from $(x, y)$ to $(x+1, y)$ is open in $\widetilde{\mathbb{P}}$ if and only if $G(R)$ holds in $\mathbb{P}_{p}$ for the $3 n$ by $n$ rectangle $[2 n x, 2 n x+3 n] \times[2 n y, 2 n y+n]$. Similarly, the edge from $(x, y)$ to $(x, y+1)$ is open in $\widetilde{\mathbb{P}}$ if and only if $G\left(R^{\prime}\right)$ holds in $\mathbb{P}_{p}$ for the $n$ by $3 n$ rectangle $[2 n x, 2 n x+n] \times[2 n y, 2 n y+3 n]$.
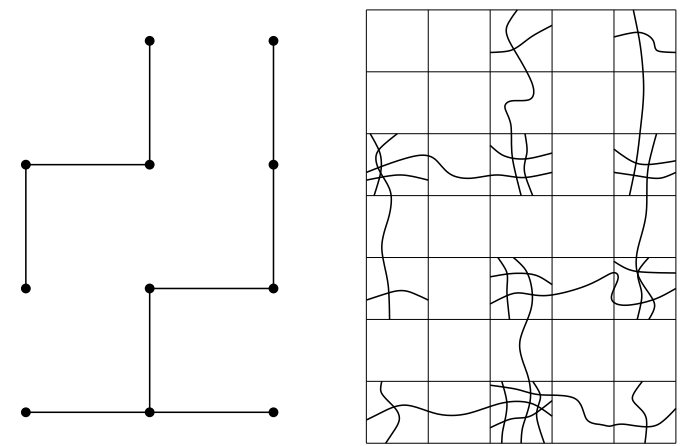

Figure 9: A set of open edges in $\widetilde{\mathbb{P}}$ (left), and corresponding rectangles $R$ drawn with $G(R)$ holding in $\mathbb{P}_{p}$.

This probability measure is indeed 1-dependent, as $G(R)$ depends only on the states of edges in $R$, and vertex disjoint edges of $\mathbb{Z}^{2}$ correspond to disjoint rectangles.

By Lemma 12] $\widetilde{\mathbb{P}}\left(\left|C_{0}\right|=\infty\right)>0$. However, we have defined $G(R)$ in such a way that a $\widetilde{\mathbb{P}}$-open path guarantees a corresponding (much longer) open path 
in the original bond percolation, using the fact that horizontal and vertical crossings of a square must meet; see Figure 9 Hence, $\mathbb{P}_{p}\left(E_{\infty}\right) \geq \widetilde{\mathbb{P}}\left(\left|C_{0}\right|=\right.$ $\infty)>0$, completing the proof of Theorem 10

The argument above works with 2 by 1 rectangles, using internal crossings as in the second proof of Theorem [10] Also, it is enough to require a vertical crossing of the left-hand end square of each rectangle $R$, and a horizontal crossing of the bottom square of each $R^{\prime}$. Appealing to $s_{n} \geq i_{n}^{1 / 2}$, as before, to prove percolation it thus suffices to find an $n$ with $i_{n}^{3 / 2} \geq p_{0}$, where $p_{0}$ is a constant for which Lemma 12 holds. Using the value $p_{0}=0.8639$ from [3], $i_{n} \geq 0.907 \ldots$ will do.

\section{Extensions}

The arguments above give short proofs of Kesten's Theorem, using Theorem 2 as a key ingredient. In fact, as we shall show in future work [5], these arguments, like those of Harris and Kesten, easily give further results. For example, our method gives exponential decay of $\left|C_{0}\right|$ when $p<1 / 2$, showing that $p_{T}$, the critical probability at which $\mathbb{E}\left(\left|C_{0}\right|\right)$ becomes infinite, is also equal to $1 / 2$, another result of Kesten [16. Furthermore, although we have written everything for bond percolation in $\mathbb{Z}^{2}$, the same method gives similar results in other contexts; for example, we obtain new, simple proofs that $p_{T}=p_{H}$ for site percolation in the square lattice, and that both critical probabilities are equal to $1 / 2$ for site percolation in the triangular lattice. The basic method used in Section 5 is much more general, applying to many other two-dimensional contexts. Indeed, as remarked earlier, it was developed in [4 to prove that the critical probability for random Voronoi percolation in the plane is $1 / 2$.

Acknowledgements. Since the first draft of this paper was written we have had stimulating discussions with Christian Borgs, Jennifer Chayes and Geoffrey Grimmett. We are grateful to all of them.

\section{References}

[1] R. Ahlswede and D.E. Daykin, An inequality for the weights of two families of sets, their unions and intersections, Z. Wahrsch. Verw. Gebiete 43 (1978), $183-185$.

[2] M. Aizenman, J.T. Chayes, L. Chayes, J. Fröhlich and L. Russo, On a sharp transition from area law to perimeter law in a system of random surfaces, Comm. Math. Phys. 92 (1983), 19-69.

[3] P. Balister, B. Bollobás and M. Walters, Continuum percolation with steps in the square or the disc, to appear in Random Structures and Algorithms. 
[4] B. Bollobás and O. Riordan, The critical probability for random Voronoi percolation in the plane is $1 / 2$, submitted. Preprint available from http://arXiv.org/math/0410336.

[5] B. Bollobás and O. Riordan, Sharp thresholds and percolation in the plane, submitted. Preprint available from http://arXiv.org/math/0412510.

[6] J. Bourgain, J. Kahn, G. Kalai, Y. Katznelson and N. Linial, The influence of variables in product spaces, Israel J. Math. 77 (1992), 55-64.

[7] S.R. Broadbent and J.M. Hammersley, Percolation processes. I. Crystals and mazes, Proc. Cambridge Philos. Soc. 53 (1957), 629-641.

[8] J.T. Chayes and L. Chayes, Percolation and random media, in Critical phenomena, random systems, gauge theories. Part I, II (Les Houches, 1984), 1001-1142. North-Holland, Amsterdam, 1986.

[9] E. Friedgut and G. Kalai, Every monotone graph property has a sharp threshold, Proc. Amer. Math. Soc. 124 (1996), 2993-3002.

[10] G. Grimmett, Percolation, Second edition. Springer-Verlag, Berlin, 1999. xiv+444 pp. ISBN 3-540-64902-6.

[11] J.M. Hammersley, Percolation processes. II. The connective constant, Proc. Cambridge Philos. Soc. 53 (1957), 642-645.

[12] J.M. Hammersley, Percolation processes: Lower bounds for the critical probability, Ann. Math. Statist. 28 (1957), 790-795.

[13] J.M. Hammersley, Bornes supérieures de la probabilité critique dans un processus de filtration, Le calcul des probabilités et ses applications. Paris, 1520 juillet 1958, Colloques Internationaux du Centre National de la Recherche Scientifique, LXXXVII (1959), pp. 17-37.

[14] T.E. Harris, A lower bound for the critical probability in a certain percolation process, Proc. Cam. Philos. Soc. 56 (1960), 13-20.

[15] J. Kahn, G. Kalai and N. Linial, The influence of variables on boolean functions, Proc. 29-th Annual IEEE Symposium on Foundations of Computer Science (FOCS'88), 68-80, IEEE, 1988.

[16] H. Kesten, The critical probability of bond percolation on the square lattice equals 1/2, Comm. Math. Phys. 74 (1980), 41-59.

[17] H. Kesten, Percolation theory for mathematicians, Progress in Probability and Statistics 2, Birkhäuser, Boston, Mass., 1982. iv+423 pp.

[18] D.J. Kleitman, Families of non-disjoint subsets, J. Combinatorial Theory 1 (1966), 153-155. 
[19] T.M. Liggett, R.H. Schonmann and A.M. Stacey, Domination by product measures, Annals of Probability 25 (1997), 71-95.

[20] L. Russo, A note on percolation, Z. Wahrsch. Verw. Gebiete 43 (1978), $39-48$.

[21] P.D. Seymour and D.J.A. Welsh, Percolation probabilities on the square lattice, in Advances in Graph Theory (Cambridge Combinatorial Conf., Trinity College, Cambridge, 1977, ed. B. Bollobás). Ann. Discrete Math. 3 (1978), pp 227-245.

[22] M.F. Sykes and J.W. Essam, Some exact critical percolation probabilities for bond and site problems in two dimensions, Physical Review Letters 10 (1963), 3-4. 